\title{
MODULES FOR THE UNDERGROUND MINE
}

\author{
${ }^{*}$ O. Lundhede ${ }^{1}$, O. Pettersson ${ }^{1}$ \\ ${ }^{I}$ Atlas Copco Rock Drills $A B$ \\ SE-70191 Örebro, Sweden \\ (*Corresponding author: oscar.lundhede@se.atlascopco.com)
}




\title{
MODULES FOR THE UNDERGROUND MINE
}

\begin{abstract}
Today, systems and machinery usually are designed to meet only one specific customer's requirements. One reason for this is that underground automation and smart functions are often developed tightly together with a customer. This is natural, since risk and cost can be shared between the customer and the supplier. Nevertheless, when the automation product develops and the market increase this will cause problems: The highly specialized products will not fulfill the next customer's demands.

In order to cope with this problem, Atlas Copco works towards a common platform. Instead of designing solutions for specific customers, we will lift the abstraction and focus on standardized modules. These modules, or function blocks, can then be combined in a large variety of configurations. The benefits of combining modules into products are, for example: increased flexibility when options are available for many machines; easier commissioning and maintenance since less different spare parts and tools are needed; and easier operation since the user interfaces have a similar look and feel.

This paper will present the ongoing work towards standardized modules. We will discuss how our Rig Control System (RCS) allows for modular design at Atlas Copco. RCS has number of standardized modules that are used in different surface and underground mining and tunneling equipment. These modules are also used for optional products such as, drilling, radio-remote control, and telematics systems.
\end{abstract}

\section{KEYWORDS}

Automation in mining, Robotics

\section{INTRODUCTION}

Underground mining is one of the most extreme occupations from several perspectives. First of all, mining operations are carried out in very hazardous environments. Some major types of hazards are vehicle accidents, roof falls, fire, explosions, toxic gases, and floods (Yarkan, Guzelgoz, Arslan, \& Murphy, 2009). One way to increase safety is to simply move operators out of the mine and use tele-remote controlled or autonomous machinery. Although, this is not an easy task since the machines must be robust in this harsh environment.

In this article we will discuss modularity for underground mining applications. We argue that this approach will help us provide state-of-the-art products for the world market. In many cases our systems and machinery are designed to meet the requirements from one specific customer. Although, this works for the first customer, the next one might have slightly different requirements. According to literature, a mass customization strategy, using modular components could increase product variety and at the same time reduce production lead time. 


\section{Modularity Concept}

First of all, we would like to adopt a definition of the concept used throughout this text. The consideration of modularity depends on the type of product: mechanical, electrical, or software. In this text we will discuss all these aspects, and therefore we have chosen a rather general definition of module. According to (Ro, Liker, \& Fixson, 2007) the following definitions of module and system are adopted within the automotive industry:

- Module A standardized unit or component, generally having a defined function in a system; a self-contained assembly used as a component of a larger system.

- System A group of interacting elements functioning as complex whole.

For example, we can consider a vehicle's seat as a module. It is a self-contained assembly and a component of a larger system, that is, the cabin. On the other hand the brake system is not a self-contained assembly that can be built in one place and then plugged into the vehicle. Instead, it is a group of interacting elements that runs from the brake pedal to the wheels. Another example is the electrical system that is connected throughout the machine. Therefore, we need to distinguish between modules and systems, and since systems often use several modules we cannot engineer a module without considering its impact on a variety of systems.

The main advantages of using a modularized design are (Huang \& Kusiak, 1998):

- decreased production cost,

- reduced production lead time,

- increased product variety, and

- easier product diagnosis, maintenance, repair, and disposal.

Decreased production cost is due to higher production volumes when modules are used in several products. Reduced production lead time comes from the fact that already designed modules will not be designed again. Furthermore, the products will have the ability of increased variety when options will be available in several different products. Finally, the products will be easier to maintain and repair when service technicians do not have to learn several different solutions to similar problems. The mentioned advantage will also increase safety for operators and service technicians, when products are handled in the same way.

In recent work (Danese \& Filippini, 2010) some of the above mentioned advantages have been empirically proven. In a study involving 201 industries, operating in machinery, electronic and manufacturing, they prove that production time is decreased and product performance increase when the modularity concept is adopted. They also emphasize the importance of a close cooperation with suppliers when these are responsible for module development. 
Although, we have to keep in mind that composition into modules must be addressed early in the design process. Some authors, including (Ulrich \& Eppinger, 1995), argue that the time reduction in design and test phases can be offset by work required in the phases of concept development and system-level design. In fact, in modular approaches component interfaces, standards and protocols must be carefully defined.

\section{Success Stories from Industry}

Using modules for rationalizing manufacturing and increasing product variety is not new. Actually, modularity is becoming a focus of attention and frequently stated as a goal of good design practice. The rest of this section will describe a number of success stories from industries within the: mechanics, electronics, and software domain.

Within the automotive business, that is mechanics domain, Scania was an early adopter of the modularity concept. According to (Kratochvíl \& Carson, 2005) the rise of their modular system during the 1950's was the main reason for their export success. Since the mid nineties Scania has intensified module sharing between truck and bus designs. For example, $85 \%$ of chassis modules can be shared. Today, many automotive suppliers, including Toyota, and Volkswagen, have followed Scania with this approach.

Dell Corporation has a success story within the electronics domain. A successful mass customization strategy, incorporating the use of modular components and clever use of webbased configuration to order, is credited for the company's success (Ro, Liker, \& Fixson, 2007). This strategy allowed Dell to compete on high volumes, low cost, and speedy deliveries, and still maintain high quality. Modular approaches for electronics are also used within the defense industry. For example, the Saab Group has developed cost-effective products for maritime security with the help of reusable modules (Pettersson, 2009).

Finally, software modules have been proven successful within robotics. Decomposition into software modules with standardized interfaces was introduced in (Brooks, Kaupp, Makarenko, Williams, \& Oreback, 2005). A further development of this is the Robotics Operating System (ROS) that has become the de facto standard architecture for robotic research (Quigley, o.a., 2009). The reusable software modules are used in a large variety of intelligent robots including the Google car.

\section{MODULES FOR MONITORING AND CONTROL}

This section will present the ongoing work towards standardized modules at Atlas Copco. So far we have adopted the modularity concept for our Rig Control System (RCS). RCS that is a computerized control system for drilling rigs, loaders and trucks was introduced by Atlas Copco in 1998. Since then a number of reusable modules have been designed and system performance has evolved over time. In 2013 the $5^{\text {th }}$ generation of the system is offered to the market. Today, more than 3000 machines equipped with RCS are in operation. Figure 1 shows how a standardized set of modules support several machine types including drill rigs, loaders, and trucks. In the center of the figure our computer modules, operator controls, and displays are shown. 


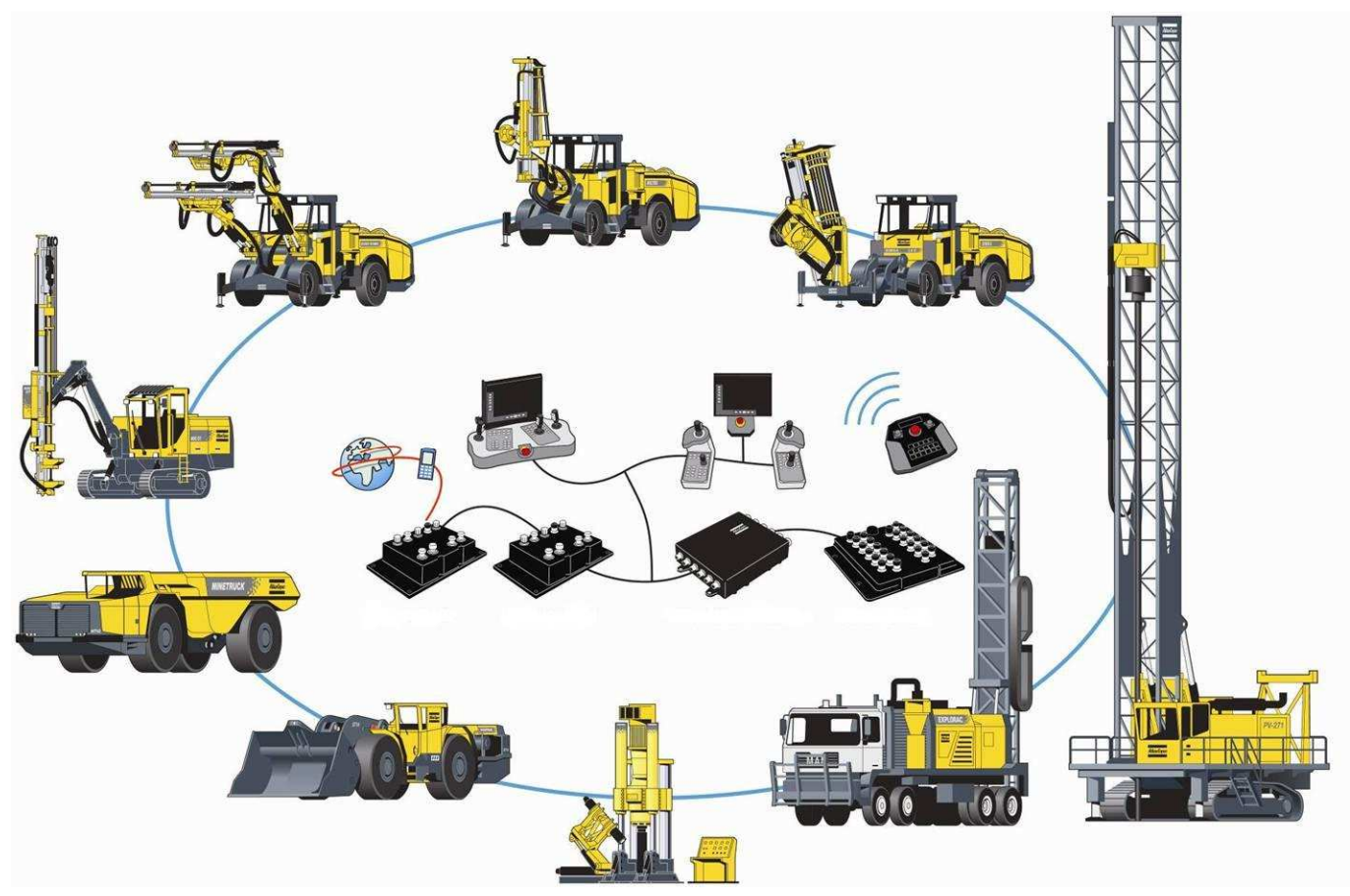

Figure 1 The rig control system (RCS) uses a standardized set of modules in several machines.

In the following we will discuss RCS modules with from mechanical, electrical, and software aspects.

\section{Mechanical Modules}

From a mechanical perspective the modules housing is central. For example, RCS has computers and displays using the same footprint and hole pattern. The connectors to cases are also standardized. Therefore, cabling is easier with standardized looms. Furthermore, maintenance is easier since modules could be exchanged without changing the surrounding parts on the machine. The modules must fulfill extreme environmental requirements in terms of temperature range, humidity, and vibration. In order to maintain quality we have chosen to design the cases in house in close cooperation with our suppliers. Figure 2 shows our ruggedized touch display. 


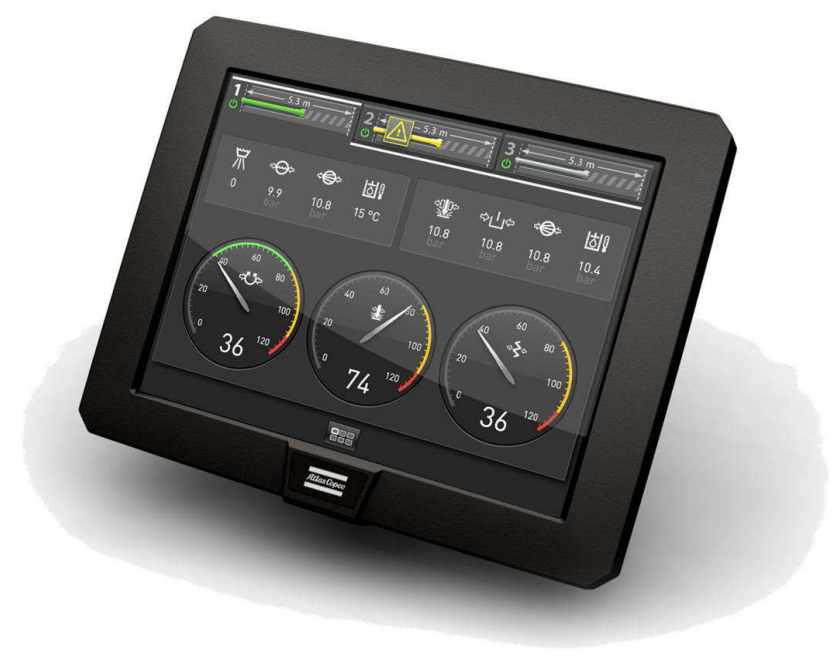

Figure 2 A 15" ruggedized touch display showing the graphical user interface of RCS5.

Another important aspect is operator ergonomics. Good mechanical design of operator seats, displays, joysticks and so on will prevent the operator from getting fatigue. Finally, the mechanical design also plays a central role in company branding. The customer should easily recognize spare parts from Atlas Copco.

\section{Electrical Modules}

Although the cases of some operator control units differ between different machines, the electrical modules inside are most likely the same. From the electrical perspective we have identified a number of modules including CPU boards and communication units that are reused in many different applications. Our electrical modules use the CAN bus for real time communication between modules in the system. For more data intensive communication we also use Ethernet. A large number of sensors are needed on the computerized machines. We try to minimize the number of different sensors in order to keep maintenance easy.

\section{Software Modules}

Software modules are abstract and therefore somewhat harder to understand. Software modules could be formalized as function blocks responsible for a certain task. Examples of such reusable software modules include: drilling, boom positioning, pump control, tele-remote control, and navigation. We also share basic software modules for: CAN-, Ethernet-, and WiFicommunication.

From RCS5 (shown in Figure 2) and onwards we use a standardized look and feel of the graphical user interface (GUI) on our machines. The idea behind this is that the operator always should feel comfortable and easy recognize the work flow when using an Atlas Copco machine. 
Communication to third part systems needs standardized protocols. Atlas Copco was one of the initiators to the International Rock Excavation Data exchange Standard (IREDES) (Chen, 2008). This standard is used for data transmission between a drill rig and other mining equipment. Today, all Atlas Copco surface drilling equipment are IREDES compliant (Appelgren, Eriksson, \& Penn, 2012).

\section{MAKING PRODUCTS OF MODULES}

Finally, we describe how our general modules are combined into a number of products suitable for several different machine types. The Radio Remote Control product, including the $\mathrm{OU}$ and MU and the Remote Monitoring product, with its DCCM and LBO are further described below. Both these products include standard RCS hardware and software designed for its applications.

\section{The Radio Remote Control}

The Radio Remote Control (RRC) system is used on several types of underground loaders and drilling rigs. The radio remote system is used to control a task from a remote location that cannot be handled from the rig or loader due to hazardous safety risk for the operator. The task that the operator needs to control depends on the application itself but the system to enable these tasks is the same.

\section{The Operator Unit.}

The Operator Unit (OU) is the module that the operator carries to control different tasks. Depending on what tasks to perform buttons and joystick configuration could vary. In the device it self we find a RCS Decoder and radio card that also are common in other systems within Atlas Copco. Operator ergonomics and safety are key drivers and something that becomes common when utilizing this operating panel.

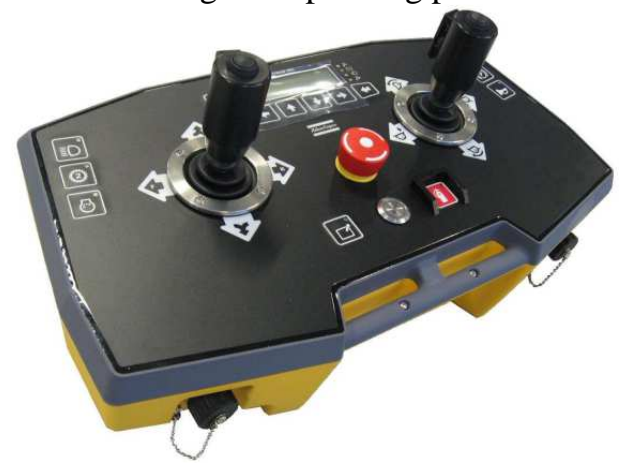

Figure 3 The ergonomic Operator Unit. 


\section{The Machine Unit}

The Machine Unit (MU) is the module that is installed on the rig or loader to interact with functions that should be controlled and monitored. The different types of rigs and loaders that could be controlled are either designed upon the Rig Control System (RCS) or on simple Direct Control System (DCS) systems. For DCS rigs and loaders the MU contains of a I/O module. This module is the direct link with the functions to be controlled. An analogue interface of both hydraulic controls and vehicle functions is pre-defined and common on all loaders (figure $4)$.

For RCS rigs and loaders the RCS I/O module is not required. The MU then contains of a radio module and a decoder only. This is connected directly to the RCS CAN network on the rig or loader. Depending on what functions that can be controlled on the equipment by RCS the same functions can be controlled from the Operator Unit.

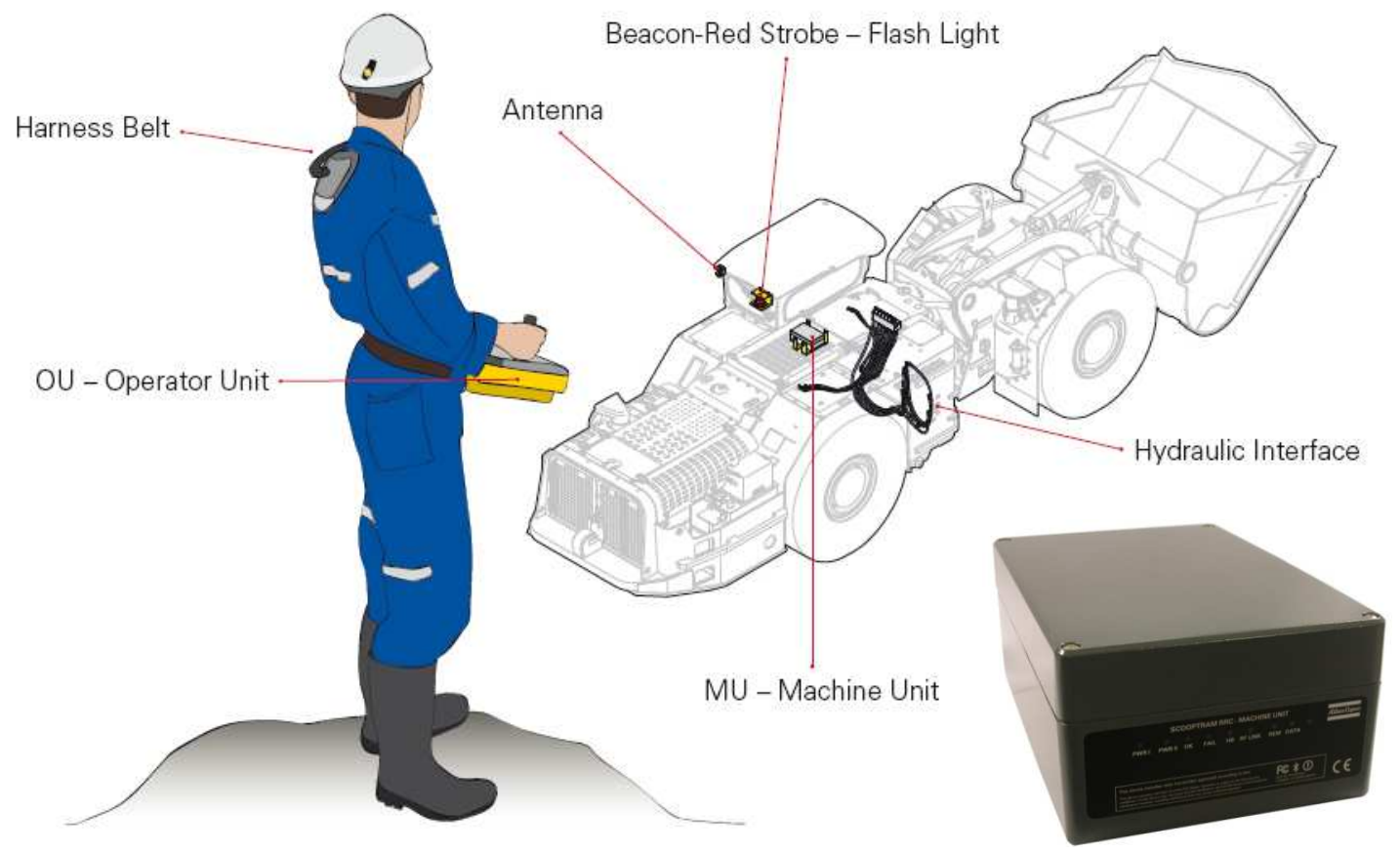

Figure 4 The Machine Unit and all necessary components on a DCS loader. 


\section{Remote Monitoring}

The Remote Monitoring product offers productivity and maintenance monitoring capabilities from any Atlas Copco rig or vehicle. It has been designed as a common product to supervise a fleet of underground or surface equipment delivered by Atlas Copco. The monitoring will enable better and more accurate service and support for the customer. At the same time the data also can be used for the customer to better supervise and manage the fleet of equipment by utilizing the data provided.

\section{The Data Collection and Communication Module}

The Data Collection and Communication Module (DCCM) is an RCS module that is used, if requested, on any type of Atlas Copco equipment for underground. The module can collect analogue, digital or CAN signals available on the equipment to be monitored. It also includes a radio module that communicates to a fixed network or portable pick up point (Mobile PUP).

\section{The Local Back Office}

The DCCM then communicates, through one of these ways, to a local back office (LBO). The protocol between the DCCM and the Local Back Office (LBO) enables that the data is stored even though no link is established. From this server the data can either be collected for maintenance support or for the customer to manage the fleet of equipment in an efficient way.

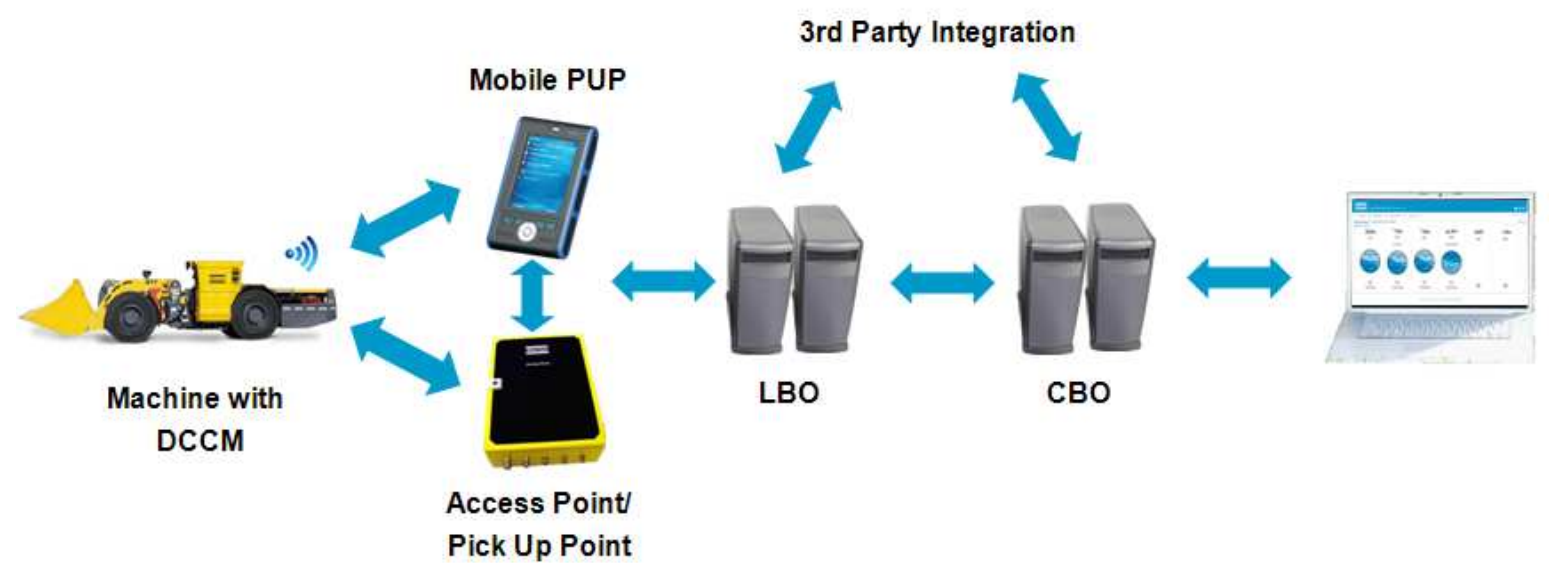

Figure 5 The Remote Monitoring system layout. 


\section{CONCLUSIONS}

This paper address that modularity is a strategic approach to product development that is based on the decomposition of the product into self-contained modules. Through this approach companies can quickly introduce new features with maintained or even increased quality. A module can be either: mechanical, electrical, or software.

A module is used together with other modules to create a function or even complete products. The module is designed to do one or several specific tasks. To become a module it must be able to be reused on several other products and applications. The functionality of a module should only include such features that can be chaired and useful for other end users. The key is to find these common requirements and demands for every module designed.

By enabling the full potential of the present common control platform we can design vehicles and products based on the same hardware and software, This will result in more functionalities and flexibilities on less numbers of equipment. Instead of designing several unique products that takes engineering hours on both design and support we can design products based on modules. By doing this we can do more with less.

\section{REFERENCES}

Appelgren, J., Eriksson, L., \& Penn, D. (2012). Rotary Drilling Automation Increasing Operator Productivity and Drilling Quality. Eighth Open Pit Operators' Conference 2012, (ss. 1318). Melbourne.

Brooks, A., Kaupp, T., Makarenko, A., Williams, S., \& Oreback, A. (2005). Towards Component-Based Robotics. IEEE International Conference on Intelligent Robots and Systems (IROS), (ss. 163-168).

Chen, G. (2008). IREDES On-line: Online Data Exchange of Automation Systems for the Mining Industry.

Danese, P., \& Filippini, R. (2010). Direct and Mediated Effects of Product Modularity on Development Time and Product Performance. IEEE International Conference on Management of Innovation and Technology (ICMIT), (ss. 438 -443).

Huang, C.-C., \& Kusiak, A. (1998). Modularity in Design of Products and Systems. IEEE Transactions on Systems, Man and Cybernetics - Part A: Systems and Humans, 28(1), 66 -77 .

Kratochvíl, M., \& Carson, C. (2005). Growing Modular: Mass Customization of Complex Products, Services and Software. Berlin: Springer.

Marshall, J., Barfoot, T., \& Larsson, J. (2008). Autonomous Underground Tramming for CenterArticulated Vehicles. Journal of Field Robotics, 25(6-7), 400-421. 
Pettersson, O. (2009). Cost-Effective Solutions for Maritime Security. Undersea Defence Technology. Cannes.

Quigley, M., Gerkey, B., Conley, K., Faust, J., Foote, T., Leibs, J., . . Ng, A. (2009). ROS: An Open-Source Robot Operating System. ICRA Workshop on Open Source Software.

Ro, Y. K., Liker, J. K., \& Fixson, S. K. (2007). Modularity as a Strategy for Supply Chain Coordination: The Case of U.S. Auto. IEEE Transactions on Engineering Management, 54(1), $172-189$.

Ulrich, K., \& Eppinger, S. (1995). Product Design and Development. New York: McGraw-Hill.

Yarkan, S., Guzelgoz, S., Arslan, H., \& Murphy, R. (2009). Underground Mine Communications: A Survey. IEEE Communications Surveys Tutorials, 11(3), 125-142. 